PROCEEDINGS OF THE

AMERICAN MATHEMATICAL SOCIETY

Volume 128, Number 11, Pages 3185-3190

S 0002-9939(00)05410-1

Article electronically published on June 7, 2000

\title{
ON THE NUMBER OF GENERATORS OF COHEN-MACAULAY IDEALS
}

\author{
CLARE D'CRUZ AND J. K. VERMA
}

(Communicated by Wolmer V. Vasconcelos)

\begin{abstract}
Several bounds on the number of generators of Cohen-Macaulay ideals known in the literature follow from a simple inequality which bounds the number of generators of such ideals in terms of mixed multiplicities. Results of Cohen and Akizuki, Abhyankar, Sally, Rees and Boratynski-Eisenbud-Rees are deduced very easily from this inequality.
\end{abstract}

\section{IntroduCtion}

The objective of this note is to present a novel approach to several results for the number of generators of Cohen-Macaulay ideals in Cohen-Macaulay local rings. Let $(R, m)$ be a Cohen-Macaulay local ring of dimension $d$. An ideal $I$ of $R$ is called a Cohen-Macaulay ideal if $R / I$ is a Cohen-Macaulay ring.

To state our main result we need to recall the basic notation for mixed multiplicities of ideals. Let $(R, m)$ be a local ring. Let $I$ be an ideal of positive height. Consider the function $C(r, s)=\ell\left(m^{r} I^{s} / m^{r+1} I^{s}\right)$. This function is given by a polynomial $Q(r, s)$ in two variables $r$ and $s$ for all large values of $r$ and $s[\mathrm{~B}$. This polynomial can be written as

$$
Q(r, s)=\sum_{i+j \leq d-1} e_{i j}\left(\begin{array}{c}
r+i \\
i
\end{array}\right)\left(\begin{array}{c}
s+j \\
j
\end{array}\right)
$$

where $e_{i j}$ are integers for all $i, j=0,1,2, \ldots, d-1$. When $i+j=d-1$, we write $e_{i+1 j}=e_{j}(m \mid I)$. These integers which appear with the monomials of degree $d-1$ in $Q(r, s)$ are nonnegative and they are called the mixed multiplicities of $m$ and $I$. Let $\mu(I)$ denote the minimum number of generators for $I$. The principal result in this paper is the following:

Theorem 1.1. Let $(R, m)$ be a Cohen-Macaulay local ring of dimension $d$. Let I be a Cohen-Macaulay ideal of $R$ of positive height $h$. Then for $q=0,1,2, \ldots, h$,

$$
\mu(I) \leq h-q+(q-1) e(R / I)+e_{h-q}(m \mid I) .
$$

Received by the editors October 7, 1998 and, in revised form, January 7, 1999.

1991 Mathematics Subject Classification. Primary 13H10, 13D40.

Key words and phrases. Cohen-Macaulay ring, Cohen-Macaulay ideal, number of generators of ideals, multiplicities, mixed multiplicities.

Presented at the first national meeting of commutative algebra and algebraic geometry held at the Institute of Astrophysics, Kodaikanal, India, March 1998.

(C)2000 American Mathematical Society 
We shall recover and generalize several results known in the literature which give upper bounds for the minimum number of generators of Cohen-Macaulay ideals. This will be done quite easily by invoking the above inequality and then applying standard results about mixed multiplicities.

\section{BOUND FOR $\mu(I)$ FOR $m$-PRIMARY IDEALS}

The proof of Theorem 1.1 is by induction on $s=\operatorname{dim} R / I$. When $s=0$, the ideal $I$ is $m$-primary. Therefore we treat this case first in this section. We begin by recalling a few facts about mixed multiplicities of ideals.

(1) Let $I$ and $J$ be $m$-primary ideals in a $d$-dimensional local ring $(R, m)$. The function $B(r, s)=\ell\left(R / I^{r} J^{s}\right)$ is called the Bhattacharya function of $I$ and $J$. Bhattacharya [B] proved that for large values of $r$ and $s$, the Bhattacharya function is given by a polynomial $P(r, s)$ of total degree $d$ in $r$ and $s$ with rational coefficients. Moreover it can be written as

$$
P(r, s)=\sum_{i+j \leq d} e_{i j}\left(\begin{array}{c}
r+i \\
i
\end{array}\right)\left(\begin{array}{c}
s+j \\
j
\end{array}\right) .
$$

The coefficients $e_{i j}$ are integers and the ones for which $i+j=d$ are positive and they are called mixed multiplicities of $I$ and $J$. We will use the notation $e_{j}(I \mid J)=e_{i j}$ for the mixed multiplicities of $I$ and $J$.

(2) Rees [R1] showed that $e_{0}(I \mid J)=e(I)$ and $e_{d}(I \mid J)=e(J)$. Here $e($.$) denotes$ the Hilbert-Samuel multiplicity.

(3) Risler and Teissier [T1] provided an interpretation of the other mixed multiplicities. They showed that the $j$ th mixed multiplicity $e_{j}(I \mid J)$ is the multiplicity of an ideal generated by $d-j$ elements of $I$ and $j$ elements of $J$ chosen sufficiently generally.

(4) Rees [R2] introduced the important concept of joint reductions of ideals which helps in calculation of mixed multiplicities. An ideal $K \subset J$ is called a reduction of $J$ if there exists an $n \in \mathbb{N}$ such that $K J^{n}=J^{n+1}\left[\mathrm{NR}\right.$. Let $I_{1}, I_{2}, \ldots, I_{d}$ be $m$-primary ideals of $R$. A set of elements $\left(x_{1}, x_{2}, \ldots, x_{d}\right)$ where $x_{i} \in I_{i}, \quad i=$ $1,2, \ldots, d$, is called a joint reduction of the set of ideals $\left(I_{1}, I_{2}, \ldots, I_{d}\right)$ if the ideal $\sum_{i=1}^{i=d} x_{i} I_{1} I_{2} \ldots I_{i-1} I_{i+1} \ldots I_{d}$ is a reduction of $I_{1} I_{2} \ldots I_{d}$. Rees showed [R3] that if $R / m$ is infinite, then joint reductions exist. The following is a crucial result in the theory of mixed multiplicities:

Theorem 2.1 (Rees's Mixed Multiplicity Theorem [R3]). Let $\left(x_{1}, x_{2}, \ldots, x_{d}\right)$ be a joint reduction of the set of ideals $(I, I, \ldots, I, J, J, \ldots, J)$ where $I$ is repeated $d-q$ times and $J$ is repeated $q$ times. Then $e_{q}(I \mid J)=e\left(\left(x_{1}, x_{2}, \ldots, x_{d}\right)\right)$.

Now we prove our main theorem for $m$-primary ideals.

Theorem 2.2. Let $(R, m)$ be a d-dimensional Cohen-Macaulay local ring and $I$ be an $m$-primary ideal of $R$. Then for $q=0,1,2, \ldots, d$,

$$
\mu(I) \leq d-q+(q-1) \ell(R / I)+e_{d-q}(m \mid I) .
$$

Proof. We may assume that $R / m$ is infinite. Let $\left(x_{1}, x_{2}, \ldots, x_{q}, a_{1}, a_{2}, \ldots, a_{d-q}\right)$ be a joint reduction of $(m, m, \ldots, m, I, I, \ldots, I)$ where $m$ is repeated $q$ times and $I$ is repeated $d-q$ times. Let $\underline{x}$ and $\underline{a}$ denote the ideals $\left(x_{1}, x_{2}, \ldots, x_{q}\right)$ and $\left(a_{1}, a_{2}, \ldots, a_{d-q}\right)$ respectively. Consider the $R$-module homomorphism

$$
\phi:(R / m)^{d-q} \oplus(R / I)^{q} \longrightarrow \frac{(\underline{x}, \underline{a})}{\underline{x} I+\underline{a} m},
$$


given by

$$
\phi\left(y_{1}^{\prime}, \ldots, y_{d-q}^{\prime}, b_{1}^{\prime}, \ldots, b_{q}^{\prime}\right)=\left(y_{1} a_{1}+\ldots+y_{d-q} a_{d-q}+b_{1} x_{1}+\ldots+b_{q} x_{q}\right)^{\prime},
$$

where primes denote the residue classes. Hence

$$
\ell\left(\frac{(\underline{x}, \underline{a})}{\underline{x} I+\underline{a} m}\right) \leq d-q+q \ell(R / I) .
$$

But

$$
\begin{aligned}
\ell\left(\frac{(\underline{x}, \underline{a})}{\underline{x} I+\underline{a} m}\right) & =\ell(R / \underline{x} I+\underline{a} m)-\ell(R /(\underline{x}+\underline{a})) . \\
& =\ell(R / I)+\ell(I / I m)+\ell(\operatorname{Im} / \underline{x} I+\underline{a} m)-e_{d-q}(m \mid I) .
\end{aligned}
$$

Hence $\mu(I) \leq d-q+(q-1) \ell(R / I)+e_{d-q}(m \mid I)$.

Corollary 2.3 (Akizuki [Ak], Cohen [C]). Let $(R, m)$ be a one-dimensional CohenMacaulay local ring. Then for any m-primary ideal $I$ of $R, \mu(I) \leq e(m)$.

Proof. Put $d=q=1$ to get $\mu(I) \leq e_{0}(m \mid I)=e(m)$.

The next result was proved by Abhyankar $[\mathrm{A}]$ for the maximal ideal.

Corollary 2.4. Let $(R, m)$ be a d-dimensional Cohen-Macaulay local ring. Let I be an m-primary ideal. Then

$$
\mu(I) \leq e(I)-\ell(R / I)+d .
$$

Proof. Put $q=0$ to get

$$
\mu(I) \leq d-\ell(R / I)+e_{d}(m \mid I)=d-\ell(R / I)+e(I) .
$$

Recall that the nilpotency degree of a nilpotent ideal $I$ is the smallest integer $t$ for which $I^{t}=0$. The next result was proved by Sally $[\underline{\mathrm{S}}]$ for $q=1$.

Corollary 2.5. Let $(R, m)$ be a d-dimensional Cohen-Macaulay local ring. Let I be an m-primary ideal. Let the nilpotency degree of $m / I$ be $t$. Then

$$
\mu(I) \leq d-q+(q-1) \ell(R / I)+t^{d-q} e(m) .
$$

Proof. It is easy to prove the following: (1) $e_{i}\left(m^{p} \mid I^{q}\right)=q^{i} p^{d-i} e_{i}(m \mid I)(2) e_{i}(I \mid I)=$ $e(I)$ and (3) for an ideal $K \subset I, e_{i}(m \mid I) \leq e_{i}(m \mid K)$. These imply that

$$
\begin{aligned}
\mu(I) & \leq d-q+(q-1) \ell(R / I)+e_{d-q}\left(m \mid m^{t}\right) \\
& =d-q+(q-1) \ell(R / I)+t^{d-q} e_{d-q}(m \mid m) \\
& =d-q+(q-1) \ell(R / I)+t^{d-q} e(m) .
\end{aligned}
$$

The next result generalizes a bound due to Boratynski, Eisenbud and Rees. This follows from Theorem 2.2 and by the following Minkowski type inequality for mixed multiplicities due to Rees and Sharp [RS] and Teissier [T2].

Theorem 2.6. Let $I$ and $J$ be m-primary ideals of a local ring $(R, m)$ of dimension $d$. Then for $i=0,1, \ldots, d$,

$$
e_{i}(I \mid J) \leq \sqrt[d]{e(I)^{d-i} e(J)^{i}} .
$$

Corollary 2.7. Let I be an m-primary ideal in a d-dimensional Cohen-Macaulay local ring. Then for $q=0,1, \ldots, d$,

$$
\mu(I) \leq d-q+(q-1) \ell(R / I)+\sqrt[d]{e(m)^{q} e(I)^{d-q}} .
$$




\section{Bound FOR $\mu(I)$ FOR COHEN-MACAulay IDEAls}

The proof of Theorem[1.1 is by induction on the dimension of $R / I$. The following lemma of Rees provides us with a tool to pass to one lower dimension.

Lemma 3.1 (Rees's Lemma [R3]). Let $(R, m)$ be a local ring with infinite residue field $R / m$. Let $\left(I_{1}, I_{2}, \ldots, I_{g}\right)$ be a set of ideals of $R$. Let $\mathcal{P}$ be a finite set of prime ideals so that none of the primes in $\mathcal{P}$ contain any of the ideals $I_{1}, I_{2}, \ldots, I_{g}$. Then there exist integers $s_{i} \geq 0$ and elements $x_{i} \in I_{i} \backslash \bigcup\{p: p \in \mathcal{P}\}$ where $i=1,2, \ldots, g$ so that for all $r_{i} \geq s_{i}$ and for all $r_{j} \geq 0, j \neq i$,

$$
x_{i} R \cap I_{1}^{r_{1}} I_{2}^{r_{2}} \ldots I_{g}^{r_{g}}=x_{i} I_{1}^{r_{1}} I_{2}^{r_{2}} \ldots I_{i}^{r_{i}-1} \ldots I_{g}^{r_{g}} .
$$

Definition 3.2. The element $x_{i} \in I_{i}$ in Rees's lemma is called superficial for the set of ideals $I_{1}, I_{2}, \ldots, I_{g}$.

Lemma $3.3(\mathrm{KV})$. Let $(R, m)$ be a local ring. Let I be an ideal of positive height $h$. If $x \in m$ is superficial for $I$ and $m$, then for $i=0, \ldots, h$,

$$
e_{i}(m \mid I)=e_{i}\left(\frac{m}{x R} \mid \frac{I+x R}{x R}\right) \text {. }
$$

Proof of Theorem 1.1. Apply induction on $s=\operatorname{dim} R / I$. If $s=0$, then $I$ is $m$ primary. Thus $\ell(R / I)=e(R / I)$. Therefore the theorem follows from Theorem 2.2. Suppose that $s \geq 1$. Then we can choose a nonzerodivisor $x \in m$ which is superficial for $m$ and $I$ and its image is a nonzerodivisor in and superficial for $m / I$. Put $\bar{R}=R / x R$ and $\bar{I}=I / x R$. Then

$$
\mu(\bar{I})=\operatorname{dim}(\bar{I} / \bar{m} \bar{I})=\operatorname{dim}\left(\frac{I+x R}{m I+x R}\right)=\operatorname{dim}\left(\frac{I}{m I+(x R \cap I)}\right)=\mu(I) .
$$

It is easy to see that $h t(\bar{I})=h t(I)$. Since $\bar{x}$ is superficial for $R / I, e(R / I)=e(\bar{R} / \bar{I})$. By Lemma $3.3 e_{h-q}(\bar{m} \mid \bar{I})=e_{h-q}(m \mid I)$. The theorem follows by induction.

Corollary 3.4. Let I be a Cohen-Macaulay ideal of positive height in a d-dimensional Cohen-Macaulay local ring $(R, m)$. Then for all $q=0,1, \ldots, h$,

$$
\mu(I) \leq h-q+(q-1) e(R / I)+e(R / I)^{h-q} e(R) .
$$

Proof. Put $s=\operatorname{dim}(R / I)$. Suppose $s=0$. Then $I$ is $m$-primary. Let the nilpotency degree of $m / I$ be $t$. Hence

$$
\begin{aligned}
e_{d-q}(m \mid I) \leq e_{d-q}\left(m \mid m^{t}\right) & =t^{d-q} e(R) \\
& \leq \ell(R / I)^{d-q} e(R) \\
& =e(R / I)^{d-q} e(R)
\end{aligned}
$$

Now let $s \geq 1$. Pick $x \in m \backslash I$ so that it is superficial for $m$ and $I$ and $\bar{x}$ is a nonzerodivisor in $R / I$ and it is superficial for $m / I$. Then

$$
\begin{aligned}
\mu(I)=\mu(\bar{I}) & \leq h-q+(q-1) e(\bar{R} / \bar{I})+e(\bar{R} / \bar{I})^{h-q} e(\bar{R}) \\
& =h-q+(q-1) e(R / I)+e(R / I)^{h-q} e(R) .
\end{aligned}
$$

Corollary 3.5 (Sally $[\mathrm{S}]$ ). $\mu(I) \leq h-1+e(R / I)^{h-1} e(R)$.

Corollary 3.6 (Rees [R4]). Suppose that $h t(I)=2$. Then $\mu(I) \leq e(R)+e(R / I)$.

Proof. Put $h=q=2$. 
Corollary 3.7 (Rees [R4]). Suppose that $h t(I)=1$. Then $\mu(I) \leq e(R)$.

Proof. Put $h=q=1$.

\section{Comparison With OTHER BOUNDS}

In this section we present some examples to show that our bounds can sometimes give better results than the previously known bounds.

First we consider a bound found by Valla in $\mathrm{V}$.

Theorem 4.1. Let $(R, m)$ be a $C M$ local ring of dimension $d$ and multiplicity $e$. Let $I$ be a CM ideal of height $h$. Suppose that $e(R / I)=\epsilon$. Put $r=\min (e, \epsilon)$. Then

a) If $h=0$, then $\mu(I) \leq e-\epsilon$.

b) If $h>0$, then $\mu(I) \leq e+\epsilon(h-1)^{2} / h+r(h-1) / h$.

c) If $h \geq 2$, and $I \subseteq m^{2}$, then $\mu(I) \leq e+\epsilon(h-1)^{2} / h+\min (r+h, \epsilon)(h-1) / h-\left(\begin{array}{l}h \\ 2\end{array}\right)$.

Let $(R, m)$ be the three-dimensional regular local ring $k[[x, y, z]]$ where $k$ is any field. Consider the ideal $I=p^{(2)}$ where $p$ is generated by the defining equations of the monomial space curve $\left(t^{3}, t^{4}, t^{5}\right)$. Then $e_{1}(m \mid I)=3$. To calculate Valla's bound notice that $e=1, \epsilon=e\left(R / p^{(2)}\right)=9$, by the associativity formula. Thus Valla's bound gives $\mu(I) \leq 6$. Our bound in Theorem 1.1 gives $\mu(I) \leq 4$. In fact $I$ is four generated. Next we consider a very appealing bound found in [DGV].

Theorem 4.2. Let $(R, m)$ be a $C M$ local ring of dimension $d \geq 1$. Let $I$ be an $m$-primary ideal such that $m^{s} \subseteq I$. Then

$$
\mu(I) \leq e(R)\left(\begin{array}{c}
s+d-2 \\
d-1
\end{array}\right)+\left(\begin{array}{c}
s+d-2 \\
d-2
\end{array}\right) .
$$

Consider the ideal $I=\left(x^{2}, x y, y^{n}\right), n \geq 2$, of the power series ring $k[[x, y]]$ over a field $k$. Then $e_{1}(m \mid I)=2$, hence the bound in Theorem 1.1 tells us that $I$ is generated by at most 3 elements. The bound in [DGV] tells us that $I$ is generated by at most $n+1$ elements. On the other hand the bound in $[\mathrm{DGV}]$ is often better for large powers of ideals. If $(R, m)$ is a regular local ring of dimension $d$, then Theorem 1.1 implies that $m^{n}$ is generated by at most $d-1+n^{d-1}$ elements. The bound in DGV] gives the exact number of generators. Hence our bound is inferior to the bound in $\overline{D G V}$ in this case.

\section{ACKNOWLEDGEMENTS}

The second author thanks Prof. W. Heinzer for useful discussions while he was visiting Purdue University and Prof. W. Vasconcelos for pointing out the references DGV] and [V].

\section{REFERENCES}

[A] S. S. Abhyankar, Local rings of high embedding dimension, Amer. J. Math. 89 (1967), 1073-1077. MR 36:3775

[Ak] Y. Akizuki, Zur Idealtheorie der einartigen Ringbereiche mit dem Teilerkettensatz, Jap. J. Math. 14 (1938), 85-102.

[B] P. B. Bhattacharya, The Hilbert function of two ideals, Proc. Camb. Philos. Soc. 53 (1957), 568-575. MR 19:727b

[BED] M. Boratynski, D. Eisenbud and D. Rees, On the number of generators of ideals in local Cohen-Macaulay rings, J. Algebra 57 (1979), 77-81. MR 82k:13021

[C] I. S. Cohen, Commutative rings with restricted minimum condition, Duke Math. J. 17 (1950), 27-42. MR 11:413g 
[DGV] L. R. Doering, Tor Gunston and W. Vasconcelos, Cohomological degrees and Hilbert functions of graded modules, Amer. J. Math. 120 (1998), 493-504. CMP 98:13

[KV] D. Katz, and J. K. Verma, Extended Rees algebras and mixed multiplicities, Math. Z. 202 (1989), 111-128. MR 90i:13024

[NR] D. G. Northcott, and D. Rees, Reductions of ideals in local rings, Proc. Cambridge Philos. Soc. 50 (1954), 145-158. MR 15:596a

[R1] D. Rees, $\mathcal{A}$ - Transforms of local rings and a theorem on multiplicities of ideals, Proc. Cambridge Philos. Soc. 57 (1961), 8-17. MR 22:9521

[R2] D. Rees, Multiplicities, Hilbert functions and degree functions, In Commutative Algebra: Durham 1981, London Mathematical Society Lecture Notes 72 (ed. R. Y. Sharp), Cambridge Univ. Press (1983), 170-178. MR 84j:13001

[R3] D. Rees, Generalizations of reductions and mixed multiplicities, J. London Math. Soc. 29 (1984), 397-414. MR 86e:13023

[R4] D. Rees, Estimates for the minimum number of generators for Cohen-Macaulay ideals , preprint.

[RS] D. Rees, and R. Y. Sharp, On a theorem of B. Teissier on multiplicities of ideals in local rings, J. London Math. Soc.(2)18 (1978), 449-463. MR 80e:13009

[S] J. D. Sally, Bounds for the number of generators of Cohen-Macaulay ideals, Pacific J. Math. 63(1976), 517-520.

[T1] B. Teissier, Cycles èvanescents, section planes, et conditions de Whitney, Singularitiés à Cargése, 1972. Astérisque 7-8 (1973), 285-362. MR 51:10682

[T2] B. Teissier, Sur une inégalité à la Minkowski pour les multiplicités, appendix to : D. Eisenbud and H. Levine, an algebraic formula for the degree of $C^{\infty}$ map-germ Ann. Math. 106 (1977), 19-44.

[V] G. Valla, Generators of ideals and multiplicities, Comm. Algebra 15(1981), 1541-1549.

SPiC Mathematical Institute, 92 G. N. Chetty Road, T. Nagar, Chennai 600017 , INDIA

E-mail address: clare@smi.ernet.in

Department of Mathematics, Indian Institute of Technology, Bombay, Powai, MumBAI 400 076, INDIA

E-mail address: jkv@math.iitb.ernet.in 